\title{
Machado-Joseph disease type 2
}

INSERM

\section{Source}

INSERM. (1999). Orphanet: an online rare disease and orphan drug data base. MachadoJoseph disease type 2. ORPHA:276241

Machado-Joseph disease type 2 is a subtype of Machado-Joseph disease (SCA3/MJD, see this term) with intermediate severity characterized by an intermediate age of onset, cerebellar ataxia and external progressive ophthalmoplegia, with variable pyramidal and extrapyramidal signs. 